\title{
Surface functionalization of dopamine coated iron oxide nanoparticles for various surface functionalities
}

Jennifer Sherwood, ${ }^{1}$ Yaolin $X u,{ }^{1}$ Kira Lovas, ${ }^{1}$ Ying Qin, ${ }^{2}$ and Yuping Bao ${ }^{1 *}$

${ }^{1}$ Chemical and Biological Engineering, The University of Alabama, Tuscaloosa, AL 35487

${ }^{2}$ Alabama Innovation and Mentoring of Entrepreneurs, The University of Alabama, Tuscaloosa, AL 35487, USA.

\begin{abstract}
We present effective conjugation of four small molecules (glutathione, cysteine, lysine, and Tris(hydroxymethyl)aminomethane) onto dopamine-coated iron oxide nanoparticles. Conjugation of these molecules could improve the surface functionality of nanoparticles for more neutral surface charge at physiological $\mathrm{pH}$ and potentially reduce non-specific adsorption of proteins to nanoparticles surfaces. The success of conjugation was evaluated with dynamic light scattering by measuring the surface charge changes and Fourier transform infrared spectroscopy for surface chemistry analysis. The stability of dopamine-coated nanoparticles and the ability of conjugated nanoparticles to reduce the formation of protein corona were evaluated by measuring the size and charge of the nanoparticles in biological medium. This facile conjugation method opens up possibilities for attaching various surface functionalities onto iron oxide nanoparticle surfaces for biomedical applications.
\end{abstract}

Keywords: Magnetic nanoparticles; Conjugation; Biomedical applications; Zwitterionic surface chemistries

*Corresponding author: electronic mail: ybao@eng.ua.edu; phone: (205) 348-9869; fax: (205) 348-7558 


\section{Introduction}

Iron oxide nanoparticles (NPs) have been widely studied for biological and biomedical applications, including targeted drug delivery[1], cell tracking[2-5], and as magnetic resonance imaging(MRI) contrastagents[6-9].For all these applications, surface functionalities of NPs are critical because they are the first encounter with biological systems. For instance, surfaces directly affect cellular uptake[10], biodistribution [11], blood circulation [12], toxicity[13], and metabolism [14].Many of these biological behaviors can be attributed to the ability of NPs attracting native proteins, also known as protein corona, and subsequently intriguing immune responses[15, 16]. Two effective surface coatings have been explored to overcome the surface effects, such asPEGylation[17] and the use ofzwitterionic molecules[18]. The dense, hydrophilic layer of the polyethylene glycol (PEG) on NP surfaces minimizes NP aggregation under physiological conditions, and, additionally, the net neutral charge of the PEGylated NPs at physiological $\mathrm{pH}$ can reduce the formation of protein corona[19]. Alternatively, zwitterionic molecule coating with neutrally charged NP surfaces is capable of repressing non-specific absorption of proteins onto NP surfaces[20]. Therefore, it highly beneficial to have a functional NP surface, which allows for easy conjugation of various surface chemistries for desirable applications.

Several conjugation methods have been studied to link various molecules onto iron oxide NP surfaces, including proteins, PEG, and other small molecules [21-28]. The use of chemical linkers to cross-link NPs and molecules has been the most explored approach[29, 30]. These linkers include carbodiimide (EDC)[31, 32],N-hydroxylsuccinimide (NHS) ester cross-linker[33], andmaleimide $[34,35]$. A general drawback for linker chemistry is the specific conjugation conditions and low conjugation efficiency, such as acidic conditions ( $\mathrm{pH}$ 4.5-5.5) for EDC, $\mathrm{pH}$ 7.2-8.0 at $4{ }^{\circ} \mathrm{C}$ for NHS. Thelow conjugation efficiency is mainly a result of completion reactions.We recently showed that dopamine-functionalized surfaces of iron oxide NPs can 
easily conjugate with protein molecules $[36,37]$, which also offers a great platform to link other molecules.

In this paper, we report the direct conjugation of various molecules onto dopamine-coated iron oxide NP surfacesvia a facile, linker-free conjugation method previously developed by our group[38]. These small molecules include glutathione (GSH), cysteine (Cys), lysine (Lys), and tris(hydroxymethyl)aminomethane (Tris), which lead to various surface functionalities after conjugation. At physiological $\mathrm{pH}$, these molecules are either zwitterionic ions or neutrally charged, which can potentially suppress the formation of the protein corona in vitro and in vivo.The success of conjugation was evaluated using Fourier transform infrared spectroscopy (FTIR) spectra. The hydrodynamic size and zeta potentials of the NPs were also measured at different pHs,and compared with dopamine-coated NPs.Stability of conjugated NPs in biological medium were testedas a function of incubation time. The variation in NP sizes and chargeswas mainly used to determine whether the conjugated NPs affect surface protein adsorption. The study provides a general platform to link various small molecules onto iron oxide NPs, which provides a set of NPs for various biological and biomedical studies.

\section{Experimental}

\subsection{Dopamine-coated nanoparticles}

The spherical iron oxide NPs were synthesized via thermal decomposition following our previously established procedures[39]. Specifically, an iron oleate precursor was decomposed in the presence of oleic acid (OA) and tryoctylphosphine oxide (TOPO) at $320^{\circ} \mathrm{C}$ for 2.5 hours. Subsequently, the hydrophobic ligands of NPs were replaced with dopamine molecules via a ligand exchange methodwith some modifications[36, 37, 39, 40]. In brief, $1 \mathrm{~mL}$ of assynthesized iron oxide NPs in chloroform $(5 \mathrm{mg} / \mathrm{mL})$ was mixed with $2 \mathrm{~mL}$ of dopamine aqueous solution (3 $\mathrm{mg} / \mathrm{mL})$. The mixture was sonicated for 5 minutes to form an emulsion, followed by the addition of $15 \mathrm{~mL}$ of acetone to facilitate phase transfer. The NPs were then separated out 
of solution via magnetic separation and washed three times with waterto remove free dopamine. The dopamine-coated NPs were then dispersed water to form a stock solution of $(1 \mathrm{mg} / \mathrm{mL})$. Before conjugation, the NP surfaces were activated with addition of $\mathrm{NaOH}$, where the $\mathrm{pH}$ increase facilitates the formation of quinone structure from the catechol groups of dopamine. The quinone structure allows for facile conjugation of molecules containing $-\mathrm{NH}_{2}$ or $-\mathrm{SH}$ groups via Schiff base or Michael's addition[38].

\subsection{Conjugation of small molecules}

Small molecule conjugation was achieved by simply mixing the activated NP solution with conjugation molecules at a 1:10 molar ratio based on the theoretical amount of dopamine on the NP surfaces. The use of excess small molecules allows for full coverage of NP surfaces with conjugating molecules. NP aqueous solution was degassed using Argon gas prior to the conjugation to prevent oxidation of some small molecules. In order to ensure maximum conjugation efficiency, NPs were activated immediately prior to conjugation with the addition of $\mathrm{NaOH}$ to adjust the $\mathrm{pH}$ to 9 . The reaction mixture of activated NPs and small molecules were incubated at $37^{\circ} \mathrm{Cfor} 12$ hours. After three washes with water, the conjugated NPs were collected for further analysis.NPs were characterized using transmission electron microscopy (TEM), dynamic light scattering (DLS), and Fourier transform infrared spectroscopy (FTIR) to verify the presence of the small molecules on the surface of the NPs.

The stability of the NPs was assessed by dispersing conjugated NPs in $10 \%$ fetal bovine serum (FBS) Eagle's minimal essential medium (EMEM) and incubated at $37^{\circ} \mathrm{Cfor}$ up to 4 hours. The 4 hour incubation was chosen based on prior reports that 4 hour incubation is requiredfor in vitro cellular uptake[13, 38]. DLS measurements (size and zeta potential) were performed at $t=0,30$ min, 2 hours, and 4 hours to determine if the NPs experienced non-specific binding of serum proteins on the NPs surfaces. 


\section{Results and Discussion}

The iron oxide NPs were synthesized via thermal decomposition at high temperature in organic solvent, which producesmonodisperse, highly crystalline NPs. Subsequently, the organic ligands were replaced with dopamine wherea thin polydopamine layer likely formed, leaving the catechol groups on the NP surface for further conjugation. Figure 1 a shows the TEM image of $12 \mathrm{~nm}$ dopamine-coated iron oxide NPs from a typical reaction. The NPs were well dispersed after ligand exchange and retained their high crystallinity (Figure 1, insert). The hydrodynamic size of the dopamine-coated NPS in solution at pH 6 from DLS was about $38 \mathrm{~nm}$ (Figure 1b), which was much larger than the core size of $12 \mathrm{~nm}$. The size increase was possibly resulted from either hydrogen bond formation between neighboring catechol groups or formation of a thin layer of polydopamine during the ligand exchange[37]. With increasing solution $\mathrm{pH}$ to 9, the NP size decreased to $31 \mathrm{~nm}$ because of deprotonation of surface functional groups, which helped to further separate NPs from each other (Figure 1b). 

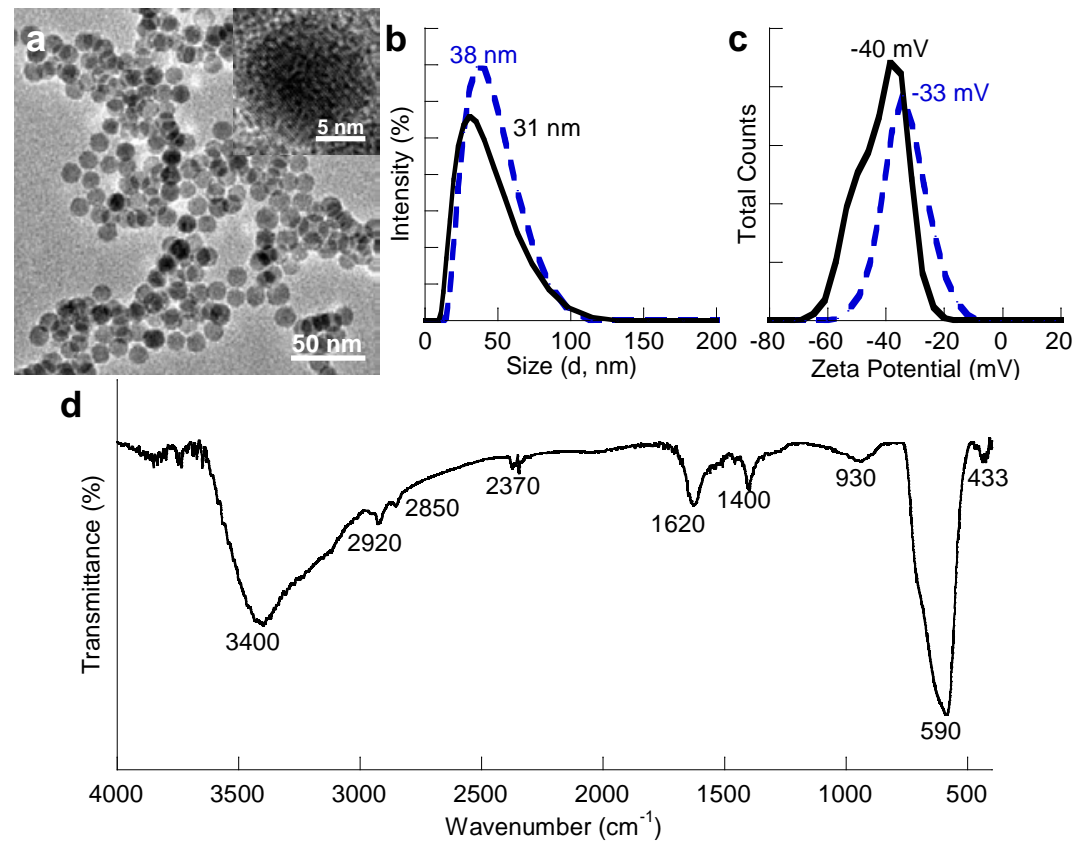

Figure 1: Dopamine-coated NPS (a) TEM and high resolution images (insert), (b) DLS plot $s$ at pH 6 (dashed blue) and 9 (black), (c) zeta potential measurements pH 6 (dashed blue) and 9 (black), and (d) FTIR spectrum after activation.

Thedopamine-coated NPs werestable at $\mathrm{pH} 9$ for long periods of time, but showed visible precipitation at $\mathrm{pH}$ below 6 . The zeta potentials of theseNPs at $\mathrm{pH} 6$ and 9 were about was-33 $\mathrm{mV}$ and $-40 \mathrm{mV}$ respectively (Figure $1 \mathrm{c}$ ). The slightly lower zeta-potential at $\mathrm{pH} 9$ is another indication of more deprotonation of catechol groups at higher $\mathrm{pH}$. However, the absolute zetapotential values of these NPs at pH 6 and 9 were both above 30, an indication of NP stability in solution.Figure 1d shows the FTIR spectra of dopamine-coated NPs after activation. The detailed analysis of the dopamine-coated NPs before and after surface activation was reported previously by our group[41]. In brief, the characteristic peak at $3400 \mathrm{~cm}^{-1}$ corresponded to the catechol of dopamine. The peaks for aromatic $\mathrm{C}=\mathrm{C}$ bonds appeared around1400 $\mathrm{cm}^{-1}$ with astrong $-\mathrm{CH}=\mathrm{CH}$ - ring breathing mode around $930 \mathrm{~cm}^{-1}$. The characteristic $-\mathrm{C}=\mathrm{O}$ band in Quinone structure at $1620 \mathrm{~cm}^{-1}$ was an indication of successful activation of dopamine molecules on NP surfaces. The lack of the typical phenol alcohol band at $1065 \mathrm{~cm}^{-1}$ was another indication 
of successful activation. The absorption bands at lower wavenumbers (590 and $433 \mathrm{~cm}^{-1}$ ) were related to Fe-O bonds in the tetrahedral and octahedralsites. The tetrahedral sites have a lower bond length with expected higher stretching frequency, where the $590 \mathrm{~cm}^{-1}$ corresponds to the intrinsic stretching vibrations of the Fe-O at the tetrahedral sites. The octahedral Fe-O stretching is normally around $400 \mathrm{~cm}^{-1}$ with a much weaker intensity[42].
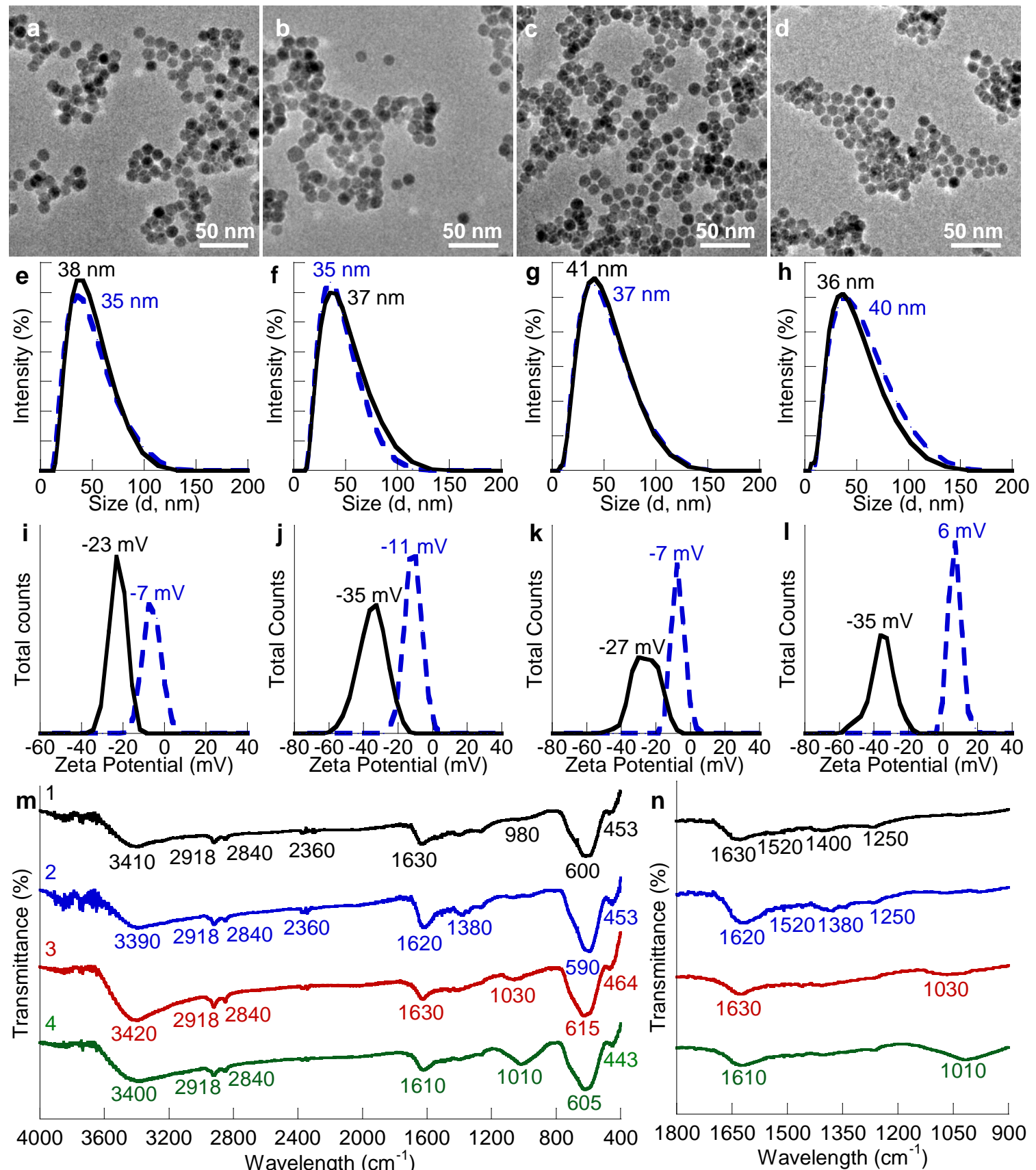

180016501500135012001050900 Wavelength $\left(\mathrm{cm}^{-1}\right)$ 
Figure 2: Conjugated NPs with GSH (a, e, i, and m1), Cys (b,f,j, and m2), Lys(c,g,k, and m3), and Tris (d,h,l, and m4). (a-d) TEM images, (e-h) DLS plots pH 6 (dashed blue) and 9 (black), (iI) zeta potential plots at $\mathrm{pH} 6$ (dashed blue) and9 (black), (m) FTIR spectra, and (n) detailed scan of FTIR spectra in the range of $900-1800 \mathrm{~cm}^{-1}$.

After conjugated with various small molecules, the size and size distribution of NPs were not affected (Figure 2a-d). In addition, the conjugated NPs were well dispersed without evident aggregations. Because of the small sizes of conjugating molecules, the hydrodynamic sizes of NPs did not show significant variations (Figure 2e-h). However, the zeta-potential of the NPs was altered greatly after conjugation (Figure $2 \mathrm{i}-\mathrm{I}$ ). Compared to the zeta-potentials of $-33 \mathrm{mV}$ and $-40 \mathrm{mV}$ at $\mathrm{pH} 6$ and 9 for dopamine-coated NPs, the zeta-potentials of conjugated NPs at pH 6 and 9 were -7 and -23 mV for GSH conjugation, -11 and -35 mVfor Cys conjugation, -7 and -27 mVfor Lys conjugation, and 6 and $-35 \mathrm{mV}$ for Tris conjugation. The changes in zetapotentials was an indication of successful conjugation. Because the activated dopamine molecules interact with amine and thiol groups, the conjugation of GSH, Cys, and Lys generated zwitterionic forms, where the surface charges change with $\mathrm{pH}$. In contrast, the conjugation of Tris, generated close to neutral surface at $\mathrm{pH}$ of 6 and more negatively charged surface at $\mathrm{pH} 9$.

The conjugation of GSH, Cys, Lys, and Tris onto dopamine-coated NP surfaces were evaluated with FTIR as shown in Figure $2 \mathrm{~m}$. Figure $2 \mathrm{n}$ is the detailed scan in the range of $900-1800 \mathrm{~cm}^{-1}$. The broad peak around $3400 \mathrm{~cm}^{-1}$ in theFTIR spectra is the characteristic $-\mathrm{OH}$ stretching in alcohols and phenols, such as $3410 \mathrm{~cm}^{-1}$ for GSH (m1),3390 $\mathrm{cm}^{-1}$ for Cys (m2), $3420 \mathrm{~cm}^{-1}$ for Lys (m3), and $3400 \mathrm{~cm}^{-1}$ for Tris(m4) respectively.Additionally, all 4 FTIR spectra showed the disappearance of the characteristic aromatic $-\mathrm{C}=\mathrm{C}$ - peaks $\left(1400 \mathrm{~cm}^{-1}\right)$ and the disappearance of thestrong $-\mathrm{CH}=\mathrm{CH}$ - ring breathing mode $\left(930 \mathrm{~cm}^{-1}\right)$, both of which were a strong presence in the FTIR of the activated dopamine coated NPs. The small, sharp but weak peaks for both GSH and Cys around $2400 \mathrm{~cm}^{-1}$ can be assigned to thiol groups, because both of them contain thiol 
groups with characteristic peaks from $2550-2600 \mathrm{~cm}^{-1}$. The disappearance and downward shift of those peaks to $2400 \mathrm{~cm}^{-1}$ indicated thiol attachment to dopamine-coated NPs. The detailed scan in the range of $900-1800 \mathrm{~cm}^{-1}$ showed characteristic $\mathrm{NH}_{2}$-Scissoring around $1500 \mathrm{~cm}^{-1}$, which were not observed in the dopamine coated NPs (Figure n). The weak broad peaks at $1250 \mathrm{~cm}^{-1}$ were likely from the $-\mathrm{C}-\mathrm{O}-\mathrm{C}$ asymmetric stretching.

In contrast, the FTIR spectra of Lys and Trisshowed no evident peaks in the $1500 \mathrm{~cm}^{-1}$ range indicating the attachment of amine groups onto NP surfaces.FTIR spectrum for Tris was similar to spectrum of dopamine-coated NPs, but without the aromatic $\mathrm{C}=\mathrm{C}$ peaks. The appearance of the characteristic C-O stretching (for $-\mathrm{OH}$ ) at $1010 \mathrm{~cm}^{-1}$ also suggested Tris attachment. FTIR spectrum for Lys conjugated NPs exhibited strong peaks at 2918 and $2840 \mathrm{~cm}^{-1}$ compared to other conjugations. These peaks were characteristic $-\mathrm{C}-\mathrm{C}$ - stretching, arising from the long $\mathrm{C}-\mathrm{C}$ side chain of Lys.
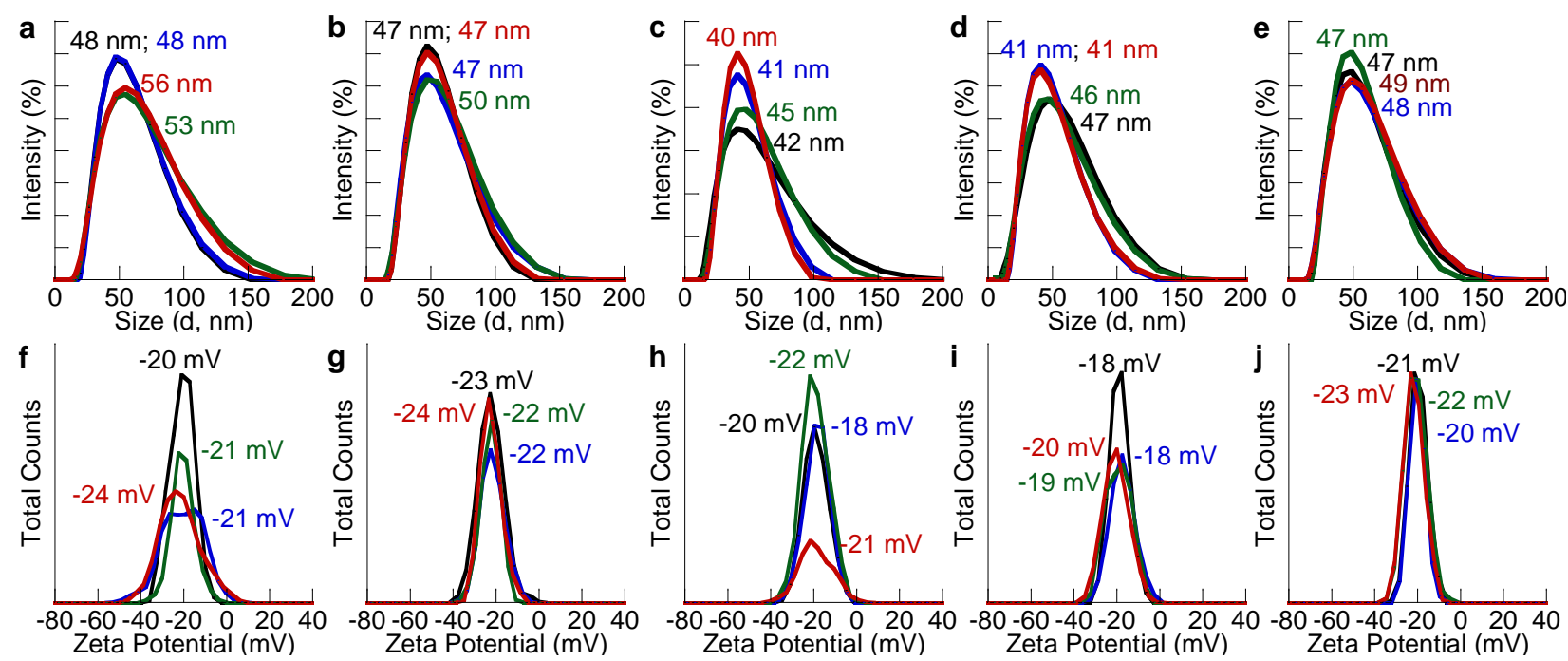

Figure 3: DLS and zeta-potential plots of dopamine-coated NPs ( $a$ and F), conjugation with GSH (b and g), Cys (c and h), Lys ( $d$ and i) and Tris (e and j) in 10\% FBS supplemented medium incubation time $\mathrm{t}=0$ (black), 30 minutes (blue), 2 hour (green), and 4 hour (red). 
The non-specific serum protein adsorption of conjugated NPs was studied in EMEM medium at physiological $\mathrm{pH}$ and compared with dopamine-coated NPS. The DLS plots of NPs were measured at incubation time of 0, 30 minutes, 2 hours and 4 hours (Figure 3). Dopaminecoated NPs showed an initial size increase from 31 to $48 \mathrm{~nm}$ and a significant drop in surface charge from -40 to $-20 \mathrm{mV}$. After 4 hour incubation, the hydrodynamic size of dopamine-coated NPs increased to $56 \mathrm{~nm}$ with an overall size increase of $25 \mathrm{~nm}$. GSH-conjugated NPs showed an initial increase in size of about $10 \mathrm{~nm}$ and maintained this size throughout the entire 4 hours. Cys-conjugated NPs experienced an initial size increase of $5 \mathrm{~nm}$. Interestingly, NPs had a reduction of size with a narrower size distribution with increasing incubation time. Only $3 \mathrm{~nm}$ size increase was observed after 4 hour incubation. Lys-conjugated NPs displayed an initial size increase of $6 \mathrm{~nm}$. Similar to the Cys-conjugated NPs, a decrease in size with narrower size distribution was observed with respect to incubation time. Tris-conjugated NPs exhibited an initial size increase of $11 \mathrm{~nm}$ and maintained that size throughout the 4 hour study. For all of the conjugated NPs, the zeta potentials were close to neutral. It was difficult to differentiate whether surface charge change resulted from protein absorption or $\mathrm{pH}$ effects, because the $\mathrm{pH}$ had a major effect on the charge of the conjugated NPs at different pHs. The hydrodynamic sizes of conjugated NPs all showed an initial size increase, but without significant variation with increasing incubation time, likely because of theZwitterionic or neutral surfaces. The proteins may have initially bound to the surfaces, but once the net charge of the ligands becameneutral, additional protein absorption was prevented. In contrast, dopamine-coated NPs showed a large increase in size and drop in zeta potential. Therefore, we believe that the conjugated NPS exhibited increased stability in physiological conditions compared to dopamine-coated NPs, which could potentially reduce non-specific protein adsorption on the NPs surfaces and potentially increase in vivo circulation time.

\section{Conclusion}


In summary, we have demonstrated effective conjugation of four amine/ thiol containing small molecules onto dopamine-coated iron oxide NP surfaces via Schiff base or Michael's addition. By attaching either GSH, Cys, Lys, or Tris to NP surfaces, different surface functionalities were achieved as confirmed by FTIR spectra. In addition, the surface charges of NPs can be adjusted with $\mathrm{pH}$ depending on the surface coatings. Importantly, the surface conjugation resulted either Zwitterionic or neutral surfaces, which increase the NP stability in solution and minimizedabsorption of serum proteins in cell. Therefore, the conjugation can potentially increase in vivo circulation time due to reduced immune response. This facile conjugation method opens up possibilities for attaching various surface functionalities onto iron oxide NP surfaces for biomedical applications.

\section{Acknowledgement}

This work was supported in part by DMR1149931. We acknowledge the UA Central Analytical Facility (CAF) and the Biological Science Department for the use of TEM.

\section{References}

[1] X.-H. Peng, X. Qian, H. Mao, A.Y. Wang, Z.G. Chen, S. Nie, D.M. Shin, Targeted magnetic iron oxide nanoparticles for tumor imaging and therapy, Int J nanomedicine, 3 (2008) 311-321.

[2] J.W.M. Bulte, T. Douglas, B. Witwer, S.-C. Zhang, E. Strable, B.K. Lewis, H. Zywicke, B. Miller, P. van Gelderen, B.M. Moskowitz, I.D. Duncan, J.A. Frank, Magnetodendrimers allow endosomal magnetic labeling and in vivo tracking of stem cells, in, Nature Biotechnology, 2001, pp. 1141-1147.

[3] J.V. Frangioni, R.J. Hajjar, In Vivo Tracking of Stem Cells for Clinical Trials in Cardiovascular Disease, in, Circulation, 2014, pp. 3378-3383.

[4] A. Taylor, A. Herrmann, D. Moss, V. Sée, K. Davies, S.R. Williams, P. Murray, Assessing the Efficacy of Nano- and Micro-Sized Magnetic Particles as Contrast Agents for MRI Cell Tracking, PLoS ONE, 9 (2014) e100259.

[5] R. Weissleder, M. Nahrendorf, M.J. Pittet, Imaging macrophages with nanoparticles, Nat Mater, 13 (2014) 125-138.

[6] B.J. McCullough, O. Kolokythas, J.H. Maki, D.E. Green, Ferumoxytol in Clinical Practice: Implications for MRI, in, Journal of Magnetic Resonance Imaging, 2013, pp. 1476-1479.

[7] P. Reimer, T. Balzer, Ferucarbotran (Resovist): a new clinically approved RES-specific contrast agent for contrast-enhanced MRI of the liver: properties, clinical development, and applications, European Radiology, 13 (2003) 1266-1276.

[8] H. Shokrollahi, Contrast agents for MRI, Materials Science and Engineering, 33 (2013) 4485-4497. 
[9] G.J. Strijkers, W.J.M. Mulder, G.A.F. van Tilborg, K. Nicolay, MRI contrast Agents: Current Status and Future Perspectives, Anti-cancer Agents in Medical Chemistry, 7 (2007).

[10] M.J.D. Clift, B. Rothen-Rutishauser, D.M. Brown, R. Duffin, K. Donaldson, L. Proudfoot, K. Guy, V. Stone, The impact of different nanoparticle surface chemistry and size on uptake and toxicity in a murine macrophage cell line, Toxicology and Applied Pharmacology, 232 (2008) 418-427.

[11] C. Chouly, D. Pouliquen, I. Lucet, J.J. Jeune, P. Jallet, Development of superparamagnetic nanoparticles for MRI: Effect of particle size, charge and surface nature on biodistribution, Journal of Microencapsulation, 13 (1996) 245-255.

[12] S.M. Moghimi, A.C. Hunter, J.C. Murray, Long-circulating and target-specific nanoparticles: Theory to practice, Pharmacological Reviews, 53 (2001) 283-318.

[13] Y. Xu, J.A. Sherwood, K.H. Lackey, Y. Qin, Y. Bao, The responses of immune cells to iron oxide nanoparticles, Journal of Applied Toxicology, 36 (2016) 543-553.

[14] O. Lunov, T. Syrovets, C. Rocker, K. Tron, G.U. Nienhaus, V. Rasche, V. Mailander, K. Landfester, T. Simmet, Lysosomal degradation of the carboxydextran shell of coated superparamagnetic iron oxide nanoparticles and the fate of professional phagocytes, Biomaterials, 31 (2010) 9015-9022.

[15] M.P. Monopoli, C. Aberg, A. Salvati, K.A. Dawson, Biomolecular coronas provide the biological identity of nanosized materials, Nature Nanotechnology, 7 (2012) 779-786.

[16] M. Lundqvist, J. Stigler, G. Elia, I. Lynch, T. Cedervall, K.A. Dawson, Nanoparticle size and surface properties determine the protein corona with possible implications for biological impacts, Proceedings of the National Academy of Sciences of the United States of America, 105 (2008) 14265-14270.

[17] E. Poselt, H. Kloust, U. Tromsdorf, M. Janschel, C. Hahn, C. Maßlo, H. Weller, Relaxivity Optimization of a PEGylated Iron-Oxide-Based Negative Magnetic Resonance Contrast Agent for $T_{2}$, in, ACS Nano, 2012, pp. 1619-1624.

[18] K.P. García, K. Zarschler, L. Barbaro, J.A. Barreto, W. O'Malley, L. Spiccia, H. Stephan, B. Graham, Zwitterionic-Coated "Stealth" Nanoparticles for Biomedical Applications: Recent Advances in Countering Biomolecular Corona Formation and Uptake by the Mononuclear Phagocyte System, Small, 10 (2014) 2516-2529.

[19] D.-F. Liu, C. Qian, Y.-L. An, D. Chang, S.-H. Ju, G.-J. Teng, Magnetic resonance imaging of postischemic blood-brain barrier damage with PEGylated iron oxide nanoparticles, Nanoscale, 6 (2014) 15161-15167.

[20] H. Wei, N. Insin, J. Lee, H.-S. Han, J.M. Cordero, W. Liu, M.G. Bawendi, Compact Zwitterion-Coated Iron Oxide Nanoparticles for Biological Applications, Nano Letters, 12 (2012) 22-25.

[21] R.A. Sperling, W.J. Parak, Surface modification, functionalization and bioconjugation of colloidal inorganic nanoparticles, Philosophical Transactions of the Royal Society a-Mathematical Physical and Engineering Sciences, 368 (2010) 1333-1383.

[22] C.G. Hadjipanayis, R. Machaidze, M. Kaluzova, L.Y. Wang, A.J. Schuette, H.W. Chen, X.Y. Wu, H. Mao, EGFRvIII Antibody-Conjugated Iron Oxide Nanoparticles for Magnetic Resonance Imaging-Guided Convection-Enhanced Delivery and Targeted Therapy of Glioblastoma, Cancer Research, 70 (2010) 63036312.

[23] M. Mahmoudi, A. Simchi, M. Imani, Recent Advances in Surface Engineering of Superparamagnetic Iron Oxide Nanoparticles for Biomedical Applications, Journal of the Iranian Chemical Society, 7 (2010) S1-S27.

[24] J. Nam, N. Won, J. Bang, H. Jin, J. Park, S. Jung, Y. Park, S. Kim, Surface engineering of inorganic nanoparticles for imaging and therapy, Advanced Drug Delivery Reviews, 65 (2013) 622-648.

[25] N. Erathodiyil, J.Y. Ying, Functionalization of Inorganic Nanoparticles for Bioimaging Applications, Accounts of Chemical Research, 44 (2011) 925-935. 
[26] J.M. Montenegro, V. Grazu, A. Sukhanova, S. Agarwal, J.M. de la Fuente, I. Nabiev, A. Greiner, W.J. Parak, Controlled antibody/(bio-) conjugation of inorganic nanoparticles for targeted delivery, Advanced Drug Delivery Reviews, 65 (2013) 677-688.

[27] W. Wei, Q.G. He, C. Hong, Surface functionalization and application for magnetic iron oxide nanoparticles, Progress in Chemistry, 20 (2008) 265-272.

[28] D. Arndt, T.M. Gesing, M. Baeumer, Surface Functionalization of Iron Oxide Nanoparticles and their Stability in Different Media, Chempluschem, 77 (2012) 576-583.

[29] J. Kalia, R.T. Raines, Advances in Bioconjugation, Current Organic Chemistry, 14 (2010) 138-147.

[30] G.T. Hermanson, Bioconjugate Techniques, 2nd Edition ed., Academic Press, 2008.

[31] C.W. Lee, K.T. Huang, P.K. Wei, Y.D. Yao, Conjugation of $y$-Fe2O3 nanoparticles with single strand oligonucleotides, Journal of Magnetism and Magnetic Materials, 304 (2006) e412-e414.

[32] F. Shamsipour, A.H. Zarnani, R. Ghods, M. Chamankhah, F. Forouzesh, S. Vafaei, A.A. Bayat, M.M. Akhondi, M. Ali Oghabian, M. Jeddi-Tehrani, Conjugation of Monoclonal Antibodies to Super Paramagnetic Iron Oxide Nanoparticles for Detection of her2/neu Antigen on Breast Cancer Cell Lines, Avicenna Journal of Medical Biotechnology, 1 (2009) 27-31.

[33] R.A. Sperling, W.J. Parak, Surface modification, functionalization and bioconjugation of colloidal inorganic nanoparticles, Philosophical Transactions of the Royal Society of London A: Mathematical, Physical and Engineering Sciences, 368 (2010) 1333-1383.

[34] L. Maurizi, H. Bisht, F. Bouyer, N. Millot, Easy Route to Functionalize Iron Oxide Nanoparticles via Long-Term Stable Thiol Groups, Langmuir, 25 (2009) 8857-8859.

[35] M. Mazur, A. Barras, V. Kuncser, A. Galatanu, V. Zaitzev, K.V. Turcheniuk, P. Woisel, J. Lyskawa, W. Laure, A. Siriwardena, R. Boukherroub, S. Szunerits, Iron oxide magnetic nanoparticles with versatile surface functions based on dopamine anchors, Nanoscale, 5 (2013) 2692-2702.

[36] Y.L. Xu, S. Palchoudhury, Y. Qin, T. Macher, Y.P. Bao, Make Conjugation Simple: A Facile Approach to Integrated Nanostructures, Langmuir, 28 (2012) 8767-8772.

[37] Y.L. Xu, D.C. Baiu, J.A. Sherwood, M.R. McElreath, Y. Qin, K.H. Lackey, M. Otto, Y.P. Bao, Linker-free conjugation and specific cell targeting of antibody functionalized iron-oxide nanoparticles, Journal of Materials Chemistry B, 2 (2014) 6198-6206.

[38] Y. Xu, D.C. Baiu, J.A. Sherwood, M.R. McElreath, Y. Qin, K.H. Lackey, M. Otto, Y. Bao, Linker-free conjugation and specific cell targeting of antibody functionalized iron-oxide nanoparticles, Journal of Materials Chemistry B, 2 (2014) 6198-6206.

[39] Y.L. Xu, Y. Qin, S. Palchoudhury, Y.P. Bao, Water-Soluble Iron Oxide Nanoparticles with High Stability and Selective Surface Functionality, Langmuir, 27 (2011) 8990-8997.

[40] A. Riedinger, P. Guardia, A. Curcio, M.A. Garcia, R. Cingolani, L. Manna, T. Pellegrino, Subnanometer Local Temperature Probing and Remotely Controlled Drug Release Based on Azo-Functionalized Iron Oxide Nanoparticles, Nano Letters, 13 (2013) 2399-2406.

[41] Y. Xu, S. Palchoudhury, Y. Qin, T. Macher, Y. Bao, Make Conjugation Simple: A Facile Approach to Integrated Nanostructures, Langmuir, 28 (2012) 8767-8772.

[42] Y. Xu, J. Sherwood, Y. Qin, R.A. Holler, Y. Bao, A general approach to the synthesis and detailed characterization of magnetic ferrite nanocubes, Nanoscale, 7 (2015) 12641-12649. 